\title{
République tchèque : mutations de l'éducation primaire et secondaire
}

Jiří Prokop, Magdalena Kohout-Diaz et Stanislav Bendl

\section{OpenEdition}

1 Journals

Édition électronique

URL : http://journals.openedition.org/ries/2144

DOI : $10.4000 /$ ries. 2144

ISSN : 2261-4265

Éditeur

Centre international d'études pédagogiques

\section{Édition imprimée}

Date de publication : 1 décembre 2011

Pagination : 28-33

ISBN : 978-2-85420-593-0

ISSN : $1254-4590$

\section{Référence électronique}

Jiří Prokop, Magdalena Kohout-Diaz et Stanislav Bendl, « République tchèque : mutations de

l'éducation primaire et secondaire », Revue internationale d'éducation de Sèvres [En ligne], 58 | décembre 2011, mis en ligne le 01 décembre 2014, consulté le 14 novembre 2019. URL : http:// journals.openedition.org/ries/2144; DOI : 10.4000/ries.2144

Ce document a été généré automatiquement le 14 novembre 2019.

(c) Tous droits réservés 


\title{
République tchèque : mutations de l'éducation primaire et secondaire
}

\author{
Jiří Prokop, Magdalena Kohout-Diaz et Stanislav Bendl
}

1 La République tchèque a été créée en janvier 1993, lorsque la République fédérale tchécoslovaque a été divisée en deux États. C'est un petit pays de longue tradition éducative, touchant les strates les plus larges de la population. Les changements politiques et économiques survenus en novembre 1989 sont un facteur déterminant pour le système éducatif actuel (Průcha, 2009). Depuis 1989, ce dernier fonctionne sur les principes de l'économie de marché et de la pluralité des partis politiques. En 1995, la République tchèque est devenue membre de l'OCDE et en 2004, membre de l'Union européenne.

2 L'administration publique a été réformée au cours des années 2000-2002 dans le sens d'une grande décentralisation. Les organismes décisifs de l'administration publique sont les différents ministères; concernant l'éducation, il s'agit du ministère de l'Éducation, de la Jeunesse et des Sports (MŠMT).

3 La République tchèque compte 10467542 habitants. Le développement démographique se distingue depuis longtemps par de grandes irrégularités ; en 2004, après des années de chute, une tendance modérément ascendante a commencé à se dessiner. En termes d'âge, $34,5 \%$ de la population appartient à la cohorte des $0-29$ ans et plus de $8 \%$ de la population (2009) est en âge d'être soumise à l'obligation scolaire. Dans la cohorte des 25-64 ans, $90 \%$ de la population a au moins une éducation secondaire et $14 \%$ une éducation supérieure (Education at a Glance, 2008).

4 La population est homogène en ce qui concerne la langue. Les minorités nationales, à l'exception de la minorité polonaise, sont extrêmement dispersées. Les Rom constituent une minorité ethnique significative. Cependant, il est impossible d'établir avec certitude leur nombre exact, qui dépend du fait de se déclarer rom ou pas. Le chiffre indiqué suite au recensement de la population de 2001 (11 746 personnes) représente un tiers du chiffre donné par le recensement précédent. Une estimation indique environ 200000 personnes. 
5 L'État est neutre du point de vue confessionnel, la liberté du culte est garantie. La religiosité de la population est l'une des plus basses en Europe. Environ $30 \%$ de la population se déclare croyante.

6 Les institutions de la République tchèque, adoptées le 16 décembre 1992, garantissent le droit à l'éducation c'est-à-dire "une éducation gratuite en école primaire et secondaire, et, suivant les compétences du citoyen et les possibilités de la société, gratuite également dans l'enseignement supérieur. »

\section{Organisation de l'enseignement}

7 L'école maternelle propose un accueil préprimaire aux enfants entre 3 et 5 ans. La scolarité obligatoire s'étend ensuite sur neuf années (entre 6 et 15 ans, niveaux d'éducation CITE 1 et $2^{1}$, premier et deuxième cycles de l'éducation de base). La plupart des enfants effectuent cette scolarité dans des écoles élémentaires (environ $80 \%$ ), subdivisées en premier degré et second degré.

8 Au début de l'enseignement secondaire existe cependant la possibilité de poursuivre la scolarité obligatoire dans des établissements parallèles, les gymnasium d'élite (elitní viceleté gymnázium), qui couvrent à la fois le premier et le second degré. La scolarité peut durer huit années (si l'on y entre en $5^{\mathrm{e}}$ classe, à l'âge de 10 ou 11 ans), six années (si l'on y entre en $7^{e}$ classe, à 12 ou 13 ans) ou quatre années (si l'on y entre en $9^{e}$ classe, à 14 ou 15 ans, à la fin de l'enseignement obligatoire), selon l'année où l'élève a réussi les examens d'entrée. Le nombre d'admis varie de $6 \%$ à $14 \%$ suivant les régions. À la fin de l'enseignement obligatoire, $96 \%$ des élèves poursuivent l'enseignement secondaire supérieur, non obligatoire :

- soit dans des gymnasium généraux, pour une durée de quatre ans (pour un peu plus de $20 \%$ de la population concernée) ;

- soit dans d'autres écoles secondaires spécialisées ( $48,5 \%$ d'élèves dans des formations de quatre ans sanctionnées par un baccalauréat, la maturita, $23 \%$ dans des formations en deux ou trois ans avec un programme spécialisé).

Ainsi, $94 \%$ des 25-34 ans ont atteint en 2008 au moins un niveau d'enseignement secondaire supérieur (CITE 3 : deuxième cycle de l'enseignement secondaire).

Les élèves qui ont obtenu le baccalauréat (plus de $72 \%$ ) peuvent ensuite poursuivre au niveau supérieur, soit dans les établissements supérieurs spécialisés (CITE 5B: enseignement universitaire à orientation professionnelle), soit dans les écoles supérieures (CITE $5 \mathrm{~A}$ et 6 : hautes écoles spécialisées et deuxième cycle de l'enseignement supérieur). Parmi les bacheliers de l'année 2008, 60 \% se sont inscrits la même année dans l'enseignement supérieur.

11 La part des écoles privées et religieuses et de leurs élèves représente en 2009-2010, pour les maternelles, $2,2 \%$ des écoles et $1,5 \%$ des élèves, pour l'élémentaire $2,6 \%$ des écoles et $1,3 \%$ des élèves, pour le secondaire $25,7 \%$ des écoles et $15,6 \%$ des élèves, pour le supérieur spécialisé $33,2 \%$ des écoles et $34,8 \%$ des étudiants et enfin $63,3 \%$ des établissements et $13,7 \%$ des étudiants pour l'enseignement supérieur. 


\section{L'éducation préscolaire}

12 L'école maternelle a une longue tradition nationale. Elle n'est pas obligatoire mais $76,5 \%$ des enfants de trois ans, $89,4 \%$ des enfants de quatre ans, et $92,8 \%$ des enfants de cinq ans la fréquentaient en 2008-2009. Les communes sont dans l'obligation de garantir à l'enfant, dont la famille est résidente (résidence principale), la scolarisation en école maternelle un an avant le début de la scolarisation obligatoire.

13 Les écoles maternelles sont en règle générale des institutions autonomes, fondées par les municipalités ou par des entreprises privées. Ces institutions assurent leur financement, à l'exception des salaires et des fournitures financés à partir du budget national. Elles peuvent solliciter une participation des parents à hauteur de $50 \%$ des coûts hors investissements; dans les écoles privées et religieuses, la participation financière à l'enseignement n'est pas limitée. La dernière année préscolaire est gratuite dans les écoles publiques.

\section{Une offre insuffisante d'écoles maternelles}

Entre 2003 et 2008, le nombre d'enfants fréquentant l'école maternelle a augmenté de plus de 15000 enfants, alors que le nombre d'écoles maternelles diminuait. Ainsi, au cours de l'année scolaire 2008-2009, 258 écoles ont disparu par rapport à l'année 2003-2004. La fermeture de certaines de ces écoles était prématurée, l'évolution démographique n'ayant pas été anticipée.

15 Si le ratio enfants/enseignante est globalement en augmentation à l'école maternelle, le nombre d'enseignantes employées au cours des trois dernières années a augmenté d'environ $1 \%$. Étant donné le nombre constant d'enfants dans les classes, fixé par décret à vingt-quatre, et la diminution du nombre d'écoles maternelles, l'augmentation du nombre d'enseignantes constitue donc sans doute une réponse à l'augmentation de la demande de scolarisation.

\section{La scolarité obligatoire}

En république tchèque, la scolarité obligatoire se fait à l'école dite " élémentaire ». Elle comporte deux degrés : le premier va de la $1^{\text {re }}$ à la $5^{\mathrm{e}}$ année (CITE 1) et le second [nižší sekundární vzděláváni] de la $6^{\mathrm{e}}$ à la $9^{\mathrm{e}}$ année (CITE 2). Les élèves dont les parents en font la demande et qui répondent aux critères d'entrée peuvent, à l'entrée de la sixième ou de la huitième année, effectuer leur scolarité obligatoire dans un gymnasium, par exemple un conservatoire, en huit ans. Il existe 4133 écoles élémentaires. Globalement, le premier et le second degré accueillent 816000 élèves.

17 En septembre 1998, l'enseignement à domicile a été validé à titre expérimental (Prokop, 2002). Depuis 2005, il est codifié par la nouvelle loi sur l'éducation en tant qu'enseignement individuel autorisé par le chef de l'établissement de référence, mais uniquement au cours du premier degré de l'école élémentaire.

Les élèves à besoins éducatifs particuliers peuvent être intégrés dans des écoles ordinaires. Si leur type de difficulté l'exige, des écoles ou éventuellement des classes avec des programmes éducatifs adaptés peuvent être créées pour ces élèves, en lien avec les programmes nationaux. 
La nouvelle loi sur l'éducation (2005) institue également une structure à deux degrés en ce qui concerne les programmes scolaires. Des programmes nationaux définissent les buts, la forme, la durée et les contenus des enseignements. À partir de ce cadre et du contexte spécifique de l'école, le directeur élabore ensuite un programme scolaire spécifique. Ce dispositif est en accord avec les évolutions européennes en faveur d'une plus grande autonomie des établissements scolaires (Bendl, 2008).

\section{Une baisse du nombre d'élèves}

Entre 2003-2004 et 2008-2009, le nombre d'élèves dans les écoles élémentaires a baissé d'environ $7 \%$ en raison d'une baisse de la démographie et, dans une moindre mesure, du départ des élèves pour les gymnasium, dont la fréquentation a augmenté.

Le nombre d'élèves par classe représente une variable fondamentale de la qualité d'un système éducatif. En 2003-2004, une classe accueillait en moyenne 20,1 élèves, alors qu'en 2008-2009 elle n'en accueillait plus que 19,2. Ceci est dû à une diminution plus rapide du nombre d'élèves que du nombre de classes à l'école élémentaire. Ce chiffre est significativement plus bas dans les écoles non publiques.

2 Entre 2001 et 2006, les heures d'enseignement ont augmenté pour toutes les catégories d'âge dans les écoles élémentaires. Les élèves âgés de 7 à 8 ans ont passé 648 heures sur les bancs de l'école en 2001, 655 heures en 2006 ; pour les élèves âgés de 9 à 11 ans, le nombre d'heures a augmenté, passant de 720 à 766 heures, et les élèves âgés de 12 à 14 ans ont étudié 892 heures en 2006 au lieu de 806 heures en 2001. Les élèves tchèques passent cependant beaucoup moins de temps à l'école que leurs camarades du même âge dans les autres pays. En 2001, pour la République tchèque, cet indicateur évoluait bien en dessous de la moyenne de l'OCDE. Au début de la décennie actuelle, le nombre moyen d'heures passées à l'école dans les pays de l'OCDE était, pour la seconde année de l'enseignement élémentaire, de 716 heures par an et pour la troisième année de 758 heures (Education at a Glance, 2009).

3 La proportion d'élèves handicapés intégrés dans les classes ordinaires a baissé.

24 Tous niveaux du système éducatif confondus, ce sont les écoles élémentaires qui intègrent le plus grand nombre d'élèves handicapés. Ceci est lié en partie au fait que certains handicaps (notamment les troubles d'apprentissage et du comportement, les troubles du langage oral, etc.) ne sont pas pris en considération dans les niveaux supérieurs de l'institution scolaire.

25 La baisse du nombre d'enfants handicapés dans les écoles élémentaires n'est cependant pas due à un affaiblissement de la volonté inclusive mais plutôt à la chute globale du nombre d'élèves de cette classe d'âge.

\section{Évaluations}

Les élèves de neuvième année de l'école élémentaire ( $15 \mathrm{ans})$ et du niveau correspondant dans les gymnasium ont été évalués par le Centre pour l'investigation des résultats de l'éducation (CERMAT) au cours des années 2005 à 2009. La finalité de cette démarche était de comparer les résultats des écoles élémentaires et des gymnasium. Les tests avaient lieu toujours au même moment et selon la même procédure, afin de garantir la rigueur de la comparaison. L'intérêt des écoles à participer à ce projet 
augmentait significativement chaque année. En 2009, deux tiers des élèves de neuvième année ont été soumis à ces tests. L'augmentation significative du nombre d'élèves participant à ces tests a confirmé l'intérêt et les besoins des écoles d'obtenir des évaluations objectives et comparables.

Les différences entre les résultats moyens ont été très significatives. Les résultats des gymnasium ont été nettement supérieurs. Cette supériorité avait été supposée mais son ampleur a surpris. Ses causes reposent d'une part sur les différences entre les deux types de population scolaire du point de vue des aptitudes ${ }^{2}$ initiales, de la motivation et d'autre part sur les différences de niveau d'exigence vis-à-vis des élèves. Néanmoins, certaines classes des écoles élémentaires atteignent les taux de réussite moyens des élèves des gymnasium.

Les analyses des résultats s'effectuent également chaque année selon le genre des élèves. Les garçons ont été plus performants que les filles au test portant sur les compétences mathématiques, et inversement les filles ont mieux réussi le test en langue tchèque. Ces résultats correspondent aux données issues d'autres recherches, données PISA comprises.

Dans les conditions d'un système éducatif décentralisé, avec un niveau d'autonomie élevé, ce type d'évaluation est un élément clé pour les différentes institutions définissant la politique éducative.

\section{L'éducation dans le second degré (st rední školy)}

L'éducation secondaire supérieure se déroule essentiellement dans des écoles du second degré qui fournissent ou un enseignement général ou un enseignement spécialisé (technique ou professionnel), avec une part toujours importante d'enseignement général.

31 La scolarisation des 15-18 ans n'est pas obligatoire mais a concerné 95,8 \% des jeunes en 2008-2009.

Selon les types d'établissements, ces études secondaires permettent donc d'obtenir :

33 La proportion des élèves inscrits dans l'enseignement secondaire est élevée depuis 2003-2004, et en augmentation au cours des dernières années. Cependant, parallèlement, la baisse du nombre d'élèves, d'enseignants et d'établissements continue et nous pouvons supposer que cette tendance se poursuivra à l'avenir, pour des raisons démographiques. Les conséquences de ces baisses ont des aspects positifs et négatifs. Le nombre d'élèves par enseignant ne cesse de baisser - aspect positif - mais le coût de l'éducation est plus élevé. Le taux le moins important d'élèves par classe se trouve dans l'enseignement secondaire (CITE $2 \mathrm{C} / 3 \mathrm{C}$, sans baccalauréat); le plus important se situe habituellement dans les classes de l'enseignement secondaire avec baccalauréat. Cela entraîne des fermetures mais aussi des regroupements d'établissements, dont le nombre a baissé de 44 entre 2006-2007 et 2008-2009. On dénombre actuellement 1438 écoles secondaires en République tchèque.

34 La demande concernant l'éducation avec baccalauréat (maturita) ne cesse de croître, alors que la part des élèves admis dans l'enseignement secondaire avec programmes spécifiques a baissé de plus de $5 \%$. L'offre d'établissements secondaires est actuellement considérée comme suffisante et variée. 
Ce qui semble le plus difficile à ce jour, c'est d'accéder aux gymnasium et aux écoles secondaires religieuses. Traditionnellement, les candidats accèdent facilement aux écoles fournissant un enseignement secondaire sans baccalauréat, le plus difficile étant de réussir les examens d'entrée des gymnasium (seul un candidat sur deux est admis). L'admission dans ces établissements s'opère à partir d'examens oraux et écrits, propres à chaque établissement et, parfois, à partir de tests d'intelligence. La décision finale est prise par le chef d'établissement à partir des compétences scolaires, officiellement critère principal de sélection. Ce dernier est cependant souverain pour décider, par exemple, d'une dispense d'examens. On peut considérer cette sélection comme précoce et vecteur d'injustice scolaire (Kohout-Diaz, 2010).

Pour l'évaluation finale des études secondaires, dont les réformes sont en cours en 2011, la proportion de disciplines optionnelles, à profil (profilové discipliny), est plus importante. L'élève choisit deux épreuves obligatoires (une en langue tchèque et, au choix, une en mathématiques, langue étrangère, sciences sociales ou encore informatique) et des épreuves optionnelles (dans un ensemble spécifique conçu par chaque établissement), avec deux niveaux de difficulté de l'épreuve. L'examen doit devenir national à l'horizon 2012 mais la responsabilité de l'organisation et de la conception de la partie optionnelle incombe au chef d'établissement.

\section{BIBLIOGRAPHIE}

Bendl S. (2008) : L'école comme institution. [Škola jako instituce.] In Bendl S., Kucharská A. (Eds) Chapitres de pédagogie et de psychologie scolaire [Kapitoly ze školní pedagogiky a školní psychologie.] Praha: PedF UK.

Education at a Glance. OECD Indicators. Paris : OCDE, 2009.

Kohout Diaz M. (2010) : Un facteur déterminant du climat à l'école élémentaire tchèque : la sélection scolaire précoce. In International Journal on Violence and Schools ( $\mathrm{n}^{\circ} 11$, septembre 2010).

Martinec L. et al. (2009) : Ce qui a changé à l'école tchèque. [Co se změnilo v českém školství.] Praha: UIV.

Prokop J. (2002) : « Le "Home schooling” dans sa forme tchèque ». [„Home schooling“ v českej podobe.] In Perspectives pédagogiques [Pedagogické rozhĺady], 2002, n 4, p. 28-32.

Prucha J. (2009) : Le système scolaire actuel en République Tchèque. [Současný školský systém v ČR.] In PRUCHA, J. Encyclopédie pédagogique. [Pedagogická encyklopedie.] Praha: Portál.

\section{NOTES}

1. CITE : Classification internationale type de l'éduca-tion, voir: http://www.uis.unesco.org/ Library/Documents/isced97-fr.pdf/

2. Nadání, le «fait d'être doué » est couramment employé en République tchèque, tout comme talentovaný žák, pour désigner un "élève talentueux » : ces termes visent à justifier la sélection scolaire précoce pratiquée de longue date dans le pays.

Revue internationale d'éducation de Sèvres, 58 | décembre 2011 
INDEX

Palabras claves : escuela preescolar, educación de la primera infancia, enseñanza primaria, enseñanza secundaria, sistema educativo

Mots-clés : école maternelle, éducation préscolaire, enseignement primaire, enseignement secondaire, système éducatif

Index géographique : République tchèque

Keywords : nursery schools, early childhood education, primary education, secondary education, educational system

\section{AUTEURS}

JIŘÍ PROKOP

Professeur à l'Université Charles, Prague, République tchèque.

MAGDALENA KOHOUT-DIAZ

Maître de conférences en sciences de l'éducation à l'Université Montesquieu Bordeaux 4, IUFM d'Aquitaine, France.

STANISLAV BENDL

Professeur à l'Université Charles, Prague, République tchèque. 